\title{
INVESTIGATING THE IMPACT OF VARYING THE NUMBER OF DISTRIBUTED ENERGY RESOURCES ON CONTROLLING THE POWER FLOW WITHIN A MICROGRID
}

\author{
Amir Fazeli \\ Substation Automation Solutions, Smart Grid Research Centre \\ Alstom Grid Ltd, \\ Stafford, St Leonards Avenue, ST17 4LX, UK \\ amir.fazeli@alstom.com
}

\author{
Mark Sumner, C. Mark Johnson, Edward Christopher \\ Power Electronics Machines and Control Group \\ University of Nottingham \\ Nottingham, University Park, NG7 2RD, UK
}

\begin{abstract}
The electrification of heat and transport in addition to integration of intermittent renewable resources into the existing electricity network is expected to occur in near future. Such a transformation is expected to force the operation of the electricity power system at different levels to its limits and would require reinforcement of the network assets at different levels. The incorporation of active management and control within microgrids and across the low voltage distribution network is thought as a cost effective solution which would facilitate wide scale integration of the emerging distributed energy resources. However since increasing the microgrid size at a certain DER penetration level would increase the total dispatchable power it is expected to affect the effectiveness of any control algorithm that operates at that level. This paper presents the findings obtained from of an investigation into the relationship between microgrid size and the effectiveness of a deterministic control algorithm implemented at that level.
\end{abstract}

Index Terms-Microgrids, Distributed Energy Resources, Power Flow Management, Demand Side Management

\section{INTRODUCTION}

$\mathrm{T}$

The electrification of heat and transport is expected to occur in the near future, which would consequently result in a substantial increase in electricity demand [1]. The increasing uptake of electric vehicles is expected to have a number of negative effects for the radial low voltage distribution network[2]. In particular a significant increase of voltage drop at the consumer end of LV feeders is expected. The integration of different forms of Distributed Generation (DG) to the low voltage network is also on the rise as different countries have set various targets to increase their share of renewable generation capacity. Large scale integration of Distributed Generation (DG) units requires a different approach to the planning and operation of the distribution grid. The foreseen adverse effects for the distribution network include voltage control, power quality, protection system and grid losses [3]. In order to investigate the impact of increasing the penetration level of the aforementioned Distributed Energy Resources (DER) within the low voltage network, the following stochastic DER, load and DG models have been created in Matlab/Simulink with a temporal resolution of one second:

- Domestic electricity load [4]

- Electric vehicle charging

- Domestic heating load [5]

- Ground source heat pump [6]

- Photovoltaic generation [7]

- Wind power generation [8]

- Microgrid battery energy storage [9]

A novel modelling technique for accurate quantification of electric vehicle charging requirements in terms of charging energy, power and duration has been developed by the authors. This modelling approach will be described in a future publication. All of these models are then used to develop a novel deterministic control algorithm (CPFC) for regulating the power flow through real time dispatch of the DERs within a microgrid [5]. The CPFC algorithm receives a power flow target $\left(P F_{T}\right)$ from an intermediate level of a smart grid control framework and determines the correct quantity and combination of DERS to dispatch at every moment. The structure and the logic behind the CPFC algorithm will be presented in a future publication. As the CPFC's operation is based on real time dispatch of DERs, the "total available DER power" is an important parameter which could determine the effectiveness of this algorithm for controlling CPF at its target value. Therefore this project has investigated the effectiveness of the CPFC algorithm with respect to the microgrid size (i.e. in terms of the number of dwellings with different forms of 
DERs). The simulation results obtained from that investigation has been analysed and presented in this paper.

\section{MICROGRID SIZE THRESHOLD}

\section{A. Expected variation of control effectiveness with Microgrid size}

As mentioned in the previous section the effectiveness of the CPFC algorithm highly depends on the total capacity of available DERs for dispatch in addition to the flexibility and storage capacity of every DER. For a given DER penetration percentage, increasing the number of dwellings within a Microgrid would entail an increase in total dispatchable DER power. This in turn is expected to enhance the control capability of the CPFC algorithm. On the other hand since the majority of these DERs are electrical loads (with an inherent storage element) and not pure sources of energy generation or energy storage, increasing the number of dwellings to include more of these dispatchable resources would simultaneously result in an increase in the total Microgrid load, which may consequently reduce the control capability of the CPFC algorithm. In order to avoid this burden and enhance the operational capabilities of the CPFC, it is essential to scale the power flow target value, $P F_{T}$ with respect to the number of dwellings and the percentage penetration of DERs. This has been taken into account in this study, as the average power flow target calculated for a single dwelling (at different DER penetrations) is multiplied by the number of dwellings within the Microgrid. Since the electric energy and power requirements for charging electric vehicles is considerably higher than the daily average load power of a household, the aggregated EV charging power has a profound effect on the CPFC's operation and could significantly influence the effectiveness of this algorithm (as illustrated in the previous section). In order to take this into consideration, three different percentage penetration levels of $30 \%, 60 \%$ and $90 \%$ for the electric vehicle charging has been set out and examined in this study. The percentage penetration of the remaining DERs was however kept constant at 50\% for different Microgrid sizes, in order to merely investigate the effect of increasing the Microgrid size and EV penetration on the operation of the $\mathrm{CPFC}$ algorithm. Investigating this question required creating different Microgrid sizes. Therefore 14 different Microgrid sizes comprised of four minor sizes between 2 to 8 dwellings and 10 major sizes between 10 and 100 dwellings were created. It is worth noting that expanding the load models in Simulink was a challenging and error prone process. Since Simulink is not an object orientated programming language, it inherently requires creation of multiple identifiers/tags with unique names to represent the loads and DERs in every dwelling. However since the CPFC's operation is based on real time control of Microgrid power flow, creating and simulating these models in Simulink was necessary and inevitable. It is also important to note that since domestic appliances and electric vehicle charging are modelled as stochastic loads, in order to capture an average measure of the CPFC's effectiveness, it was essential to run several simulations at every Microgrid size. The effect of variation created by stochastic load models is in particular more profound for smaller microgrids. Therefore it was necessary to perform a larger number of iterations for smaller microgrids to minimise this variation. Creating large models in Simulink and performing iterative simulations at every Microgrid size proved to be a very time consuming process and a complete simulation for all 14 microgrids and the three different $\mathrm{EV}$ penetration levels, took about 10 days on a $3.6 \mathrm{GHz}$ PC with 32GB of RAM. Having created different Microgrid sizes for the three different EV penetrations, many simulations were performed. Since increasing the Microgrid size is expected to increase DER dispatch flexibility and capacity, better CPF control is expected for larger microgrids. However since the majority of these DERs are electrical loads (with an inherent storage element) and not pure generation or energy storage units, increasing the number of dwellings to include more of these dispatchable DERs would simultaneously increase the total Microgrid load. This would consequently reduce the control capability of the CPFC. The overall CPF control effectiveness during an entire day is quantified by the value of the load factor, the load loss factor as shown in (1) and (2), in addition to the variation of the "percentage CPF reduction" and the "percentage CPF increase" using (3) and (4) respectively.

$$
\begin{gathered}
\text { Load Factor }=\frac{\frac{\sum_{t=1}^{86400} C P F}{86400}}{M A X(C P F)} \\
\text { Load Loss Factor }=\frac{\frac{\sum_{t=1}^{86400} C P F^{2}}{86400}}{M A X\left(C P F^{2}\right)} \\
\% \text { CPF Reduction }=100 \times \frac{\sum_{t=1}^{86400}\left(P F_{E_{t}}^{w / o}\right)-\sum_{t=1}^{86600}\left(P F_{E_{t}}^{w}\right)}{\sum_{t=1}^{8600}\left(P F_{E_{t}}^{w / o}\right)} \text { for } C P F_{t}^{w / o}>P F_{T}(3) \\
\% \text { CPF Increase }=100 \times\left|\frac{\sum_{t=1}^{86400}\left(P F_{E_{t}}^{w}\right)-\sum_{t=1}^{86400}\left(P F_{E_{t}}^{w / o}\right)}{\sum_{t=1}^{86400}\left(P F_{E_{t}}^{w / o}\right)}\right| \text { for } C P F_{t}^{w / o}<P F_{T}{ }^{(4)}
\end{gathered}
$$

Load factor is a measure of the average power over peak power during a given period and load loss factor is a measure of losses incurred as a result of peak power. The average percentage $\mathrm{CPF}$ reduction is a measure of the overall percentage difference between the " $P F_{E}$ with control" and " $P F_{E}$ without control" when the CPF is above the target, over a period of one day. Similarly the average percentage CPF increase is a measure of the overall difference between " $P F_{E}$ with control" and " $P F_{E}$ without control" when the CPF is below the target. Since the simulation is performed for an entire day which has 86400 seconds, the summations in (3) and (4) are performed for 86400 points. Therefore the calculated figures of merit are expected to initially increase with Microgrid size, up until the point when there is an adequate level of DER flexibility and capacity, beyond which increasing the Microgrid size would result in a saturation of these figures of merit, and no significant further improvement to the CPF control. This point would represent the recommended Microgrid size threshold. It is worth noting that the microgrids which are smaller than this threshold would 
still be benefiting from the operation of the CPFC algorithm, however at a comparatively lower extent.

\section{B. Variation of control effectiveness with Microgrid size}

Having created fourteen different Microgrid sizes for three different EV penetration levels, simulations were performed to determine the resultant behaviour. The effect of controlling the $\mathrm{CPF}$ for three of these microgrids with 2, 10 and 30 dwellings at $60 \%$ EV penetration is shown in Fig.1-3. It is worth noting that Fig.1-3 show the averaged values of CPF obtained from several simulations. Displaying the average CPF variation is expected to minimise the randomness created by the stochastic load models, and would represent the effectiveness of the CPFC algorithm more realistically. 60\% EV together with, 50\% GSHP and PV penetration means that, for instance for a Microgrid of 10 dwellings there are six EVs, five GSHP and five PV units. It is evident from Fig.1that for a Microgrid of two dwellings there are insufficient resources to dispatch and the CPFC is incapable of controlling the CPF to match the target during the entire 24 hour period.

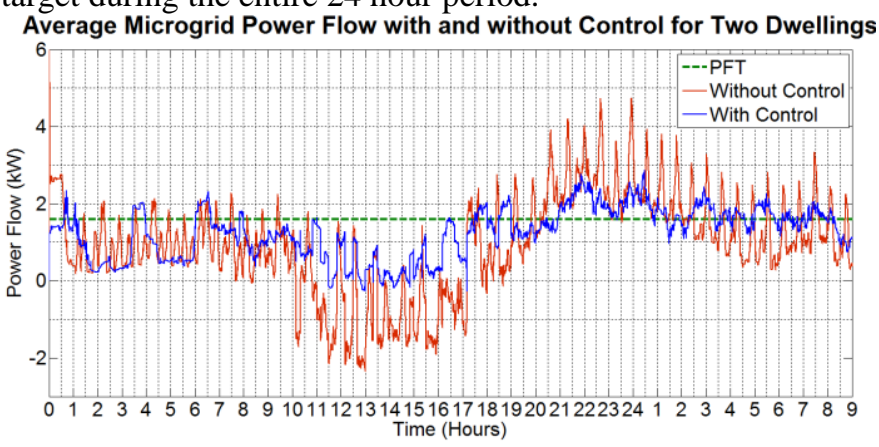

Fig.1.Microgrid power flow with and without control at $60 \%$ EV - 2 dwellings

The two remerging peaks during the interval 03:00 and 04:00 hours and interval 06:00 - 06:30 hours are created as a result of the prolonged shedding operation of the only GSHP. Since there is insufficient gap between the CPF and $P F_{T}$, it is not possible to enforce connect the only GSHP, in order to eliminate or reduce the morning peak which occurs during the interval 07:30-08:30 hours. Enforce connection of the two refrigerators with a small load power, does not seem to have any major effect on control effectiveness. The major CPF deviation from the target occurs during interval 20:30- 01:00 hours. This deviation is primarily caused when the only EV reaches the end of its maximum shedding period and starts to reconnect. Prolonged GSHP shedding also contributes to peaks during this period in addition to the ones that occur during the early morning hours of the second day. There is clearly a lack of available DERs to dispatch in this small Microgrid, in order to control the $\mathrm{CPF}$ at its target. The ineffectiveness of the CPFC is also reflected by the figures of merit presented in this section. For a Microgrid of 10 dwellings, the CPF is controlled much closer to the $P F_{T}$ as shown in Fig.2. According to this figure the majority of the deviations during the morning hours of both days are eliminated. An exception is the late evening period. The effective CPF control during the morning, midday and afternoon hours is carried out through the dispatch of the correct combination of the available DERs. This improvement to the control is also reflected by a substantial rise in the figures of merit, when the Microgrid size is increased from 2 to 10 dwellings. Despite the overall improvement in that period, a few minor CPF deviations from target still occur. For example $P F_{E}$ starts to increase during the interval 12:00 16:00 hours as there are no more resources to dispatch. The major CPF deviation from the target occurs during the early morning hours of 03:30 - 04:30 of the second day. This significant deviation is caused by the simultaneous reconnection and charging of the EVs, once they reach the end of their maximum shedding periods.

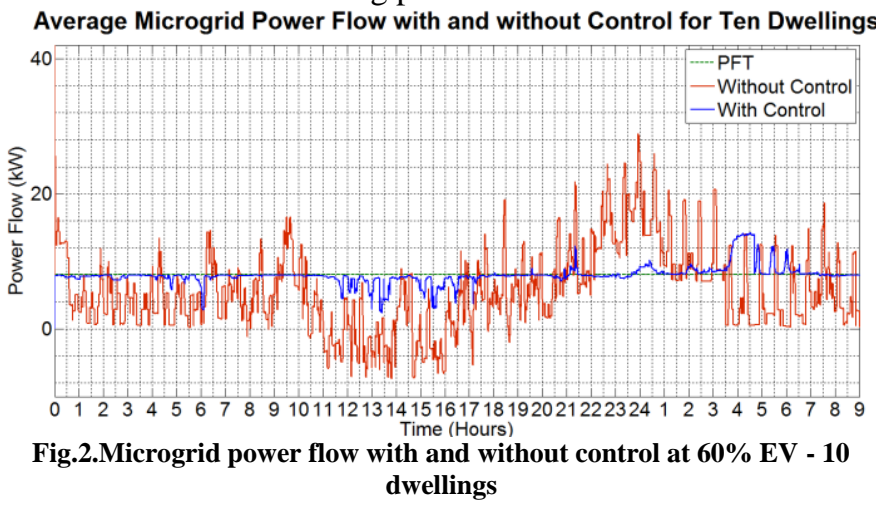

Increasing the Microgrid size to 30 dwellings results in some further improvement to control effectiveness as seen in Fig.3.

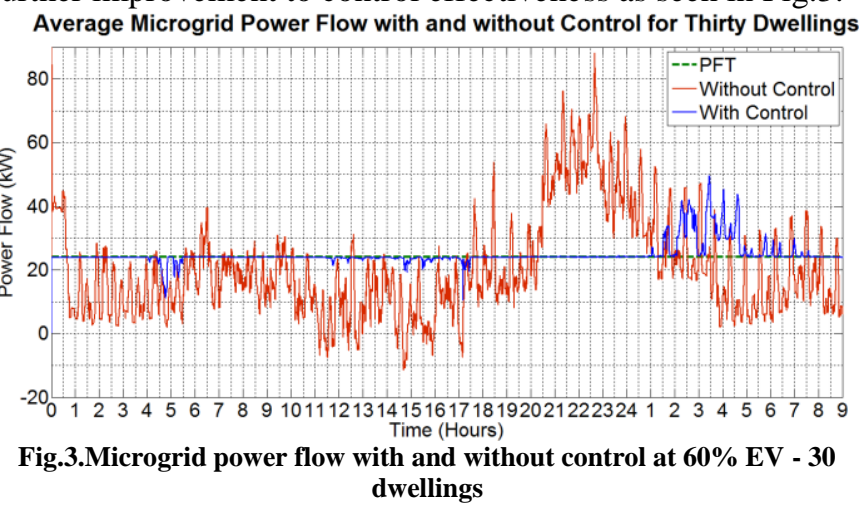

That is achieved as the majority of the minor gaps between the $\mathrm{CPF}$ and the $P F_{T}$ during morning, midday and afternoon hours of day one are mitigated. However there are exceptions during the interval 04:30 - 05:30 hours of day one when the CPFC runs out of resources to dispatch in its " $\mathrm{CPF}$ increase function". The late evening peak still remerges as a result of simultaneous EV charging. Increasing the Microgrid size beyond this point does not result in any further improvement to the CPF control and the CPF is also effectively controlled at its target value $P F_{T}$ during most of the time, for larger microgrids. This is also reflected in the values calculated for the figures of merit. The results presented in this section were obtained from repeated simulations in order to assess the effectiveness of the CPFC algorithm. This assessment is made based on average values for four different figures of merit: load factor, load loss factor, percentage CPF reduction and the percentage $\mathrm{CPF}$ increase. It is important to note that since the objective of the CPFC algorithm is to reduce the value of CPF close to the target and reduce the peak load, the value of the percentage CPF reduction is a suitable measure for assessing the effectiveness of the CPFC algorithm in this respect. On the other hand the value of percentage CPF increase gives an 
indication of the effectiveness of the CPFC algorithm at charging the storage elements of the available DERs in addition to reconnecting the loads which were previously shed. Therefore in order to distinguish between these two effects that the CPFC algorithm provides it is necessary to quantify the percentage CPF reduction and the percentage $\mathrm{CPF}$ increase as individual figures of merit. The variation of all four figures of merit with the Microgrid size at three different EV penetration levels is shown in Figs.4-7 respectively. According to [10] the annual averaged value of load factor and the load loss factor for a distribution transformer are 0.266 and 0.105 respectively. However the addition of new loads (e.g. EV and GSHP) to the network would increase the magnitude of peak power and this would consequently reduce the value of load factor. As can be seen in Fig.4, the application of the CPFC algorithm has ensured significant improvement to the value of load factor, in particular for microgrids larger than 30 dwellings.

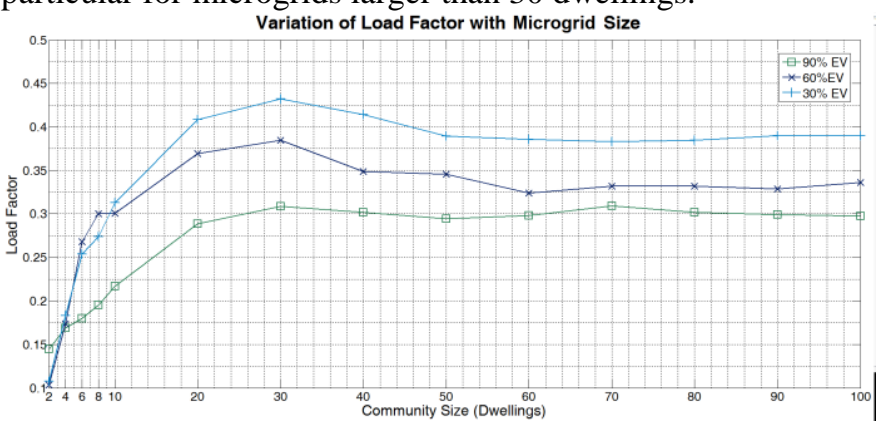

Fig.4. variation of load factor with Microgrid size for three different EV penetrations

Using the annual averaged value of load loss factor presented in [10] as a reference (0.105), it is evident in Fig.5, that the application of the CPFC algorithm has also improved the value of this figure of merit, in particular for microgrids larger than 30 dwellings, and with a lower EV penetration level.

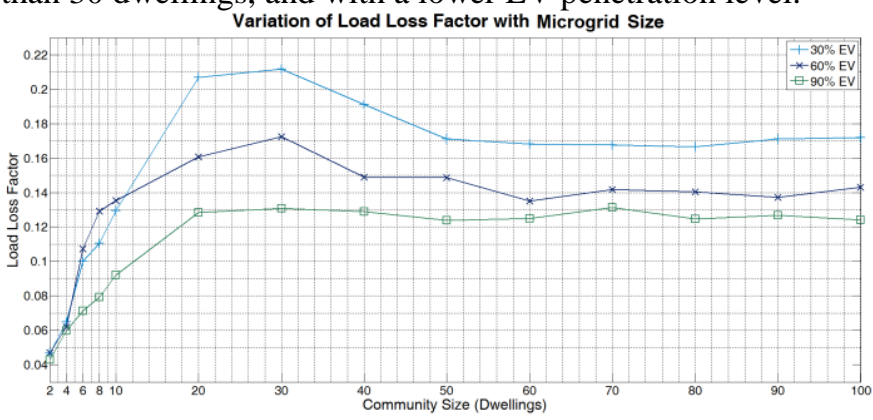

Fig.5. variation of load loss factor with Microgrid size for three different EV penetrations

As seen in Figs.4-6 the shapes of the variation of load factor, load loss factor and the percentage CPF reduction with Microgrid size are quite similar. The value of the respective figure of merit initially increases almost linearly with Microgrid size up to 10 dwellings. This significant improvement to the values of load factor and the load loss factor, are a good indication, of how the application of the CPFC algorithm within larger microgrids with more dispatchable DERs has resulted in the minimisation of both the peak power and the losses. Increasing the Microgrid size to 20 and 30 dwellings also results in a further linear increase of the respective figure of merit, however at a reduced rate. Increasing the Microgrid size above 30 dwellings, in particular at $90 \% \mathrm{EV}$ penetration, results in plateauing of all three figures of merit. According to Figs.4-7 the following two patterns commonly occur in the variation of the three figures of merit:

1) The value of the respective figure of merit for microgrids larger than 30 dwellings always reduces with EV penetration level. For example at a Microgrid of 100 dwellings, the value of load factor reduces from 0.38 to 0.33 and 0.3 as the EV penetration is increased from $30 \%$ to $60 \%$ and $90 \%$. This is an expected effect which indicates that overall the CPFC is more effective at controlling $\mathrm{CPF}$ at $P F_{T}$ when there are fewer EVs within a Microgrid.

2) The value of the respective figure of merit reduces slightly when the Microgrid size is increased from 30 to 40 dwellings. It is however interesting to note that this effect is only observed for $30 \%$ and $60 \%$ EV penetration levels.

This effect is thought to be due to insufficient diversity of the EV load in smaller microgrids. In other words the randomness introduced by the EV load is more profound for smaller microgrids with lower EV penetration. This in turn results in comparatively shorter divergence from the $P F_{T}$, and consequently a slightly higher values of figure of merit at this Microgrid size. Since the load loss factor is a measure of the overall losses and is dependent on the square of active power, the effect of insufficient diversity of EV load in microgrids smaller than 30 dwellings is more profound on the load loss factor. This explains why there is a significant drop in load loss factor when the Microgrid size is changed from 30 to 50 dwellings. Another interesting point to notice on Fig.6 is that as the EV penetration level increases the Microgrid size threshold reduces.

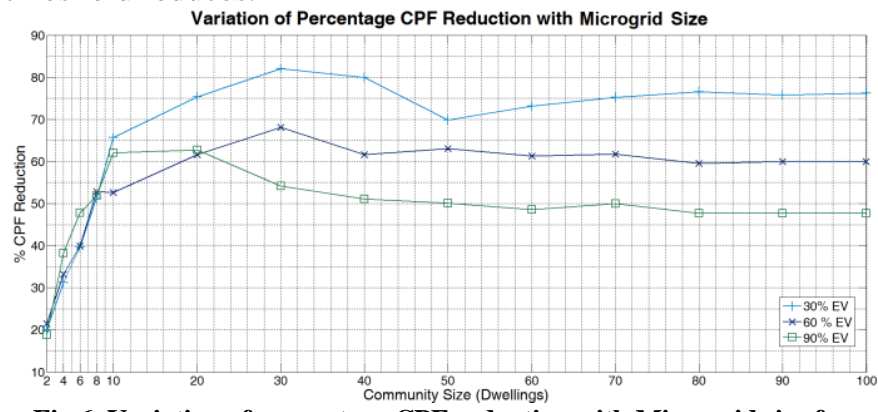

Fig.6. Variation of percentage CPF reduction with Microgrid size for three different $\mathrm{EV}$ penetrations

In other words when there are more EV to dispatch, an adequate level of flexibility can be achieved at a lower aggregation and for a smaller Microgrid. The sudden drop of percentage CPF reduction at a Microgrid of 50 dwellings with $30 \% \mathrm{EV}$ penetration is likely to be due to the randomness introduced by the EV model, at this comparatively low EV penetration level. The variation of "percentage CPF increase" with Microgrid size is shown in Fig.7. The overall trend does have the expected initial linear increase followed by a plateau for larger microgrids. It is interesting to note that despite the eventual saturation of the "percentage CPF increase" at a Microgrid of 30 dwellings, the main improvement to this figure of merit occurs when the Microgrid size is increased from 2 to 10 dwellings. This could be due to the effective execution of the "enforce connection" operation of the GSHP 
and refrigerators which only occurs when $\mathrm{CPF}$ is lower than $P F_{T}$. As adequate numbers of GSHP and refrigerator units have been allocated within every Microgrid, the value of "\%CPF increase" is quite high even for smaller microgrids.

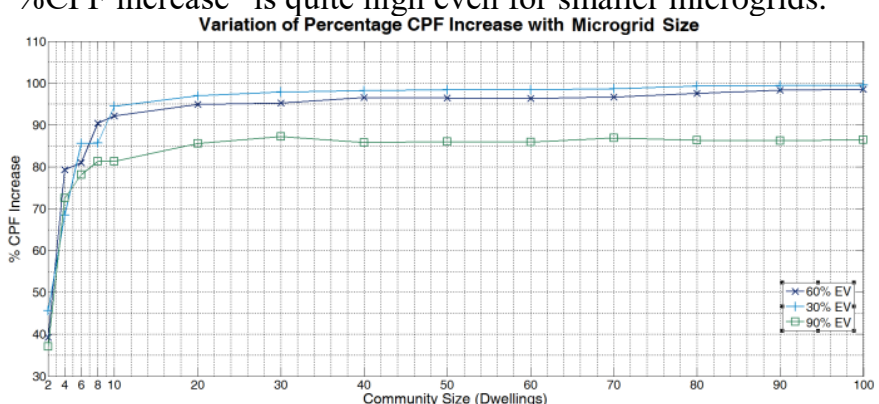

Fig.7.Variation of percentage CPF increase with Microgrid size for three different $\mathrm{EV}$ penetrations

Overall, the recommended Microgrid size threshold appears to be at about 30 dwellings as all four figures of merit starts to saturate for microgrids larger than 30 dwellings. However it is important to note that the simulation results and their analysis presented in this section are representative of the specific Microgrid described, with the given DER operational characteristics and limitations. Therefore changing the characteristic or penetration level of these DERs is expected to affect the results to some degree and might lead to some variation of the Microgrid size threshold. On the other hand the concept for determining the Microgrid size threshold is thought to be valid and its application to different microgrids with different device characteristics is expected to entail a similar trend for the variation of figures of merit with Microgrid size.

\section{CONCLUSION}

Since increasing the microgrid size at a certain DER penetration would increase the total dispatchable DER power, it is expected that the CPFC algorithm would have better control capability for larger microgrids. However as the majority of the DERs considered in this study are electrical loads (with an inherent storage element) and not pure sources of energy generation or energy storage increasing the microgrid size to include more of such dispatchable resources would simultaneously increase the total microgrid load. That would in turn reduce the control capability of the CPFC algorithm which is evaluated by number of different figures of merit. Considering both of the aforementioned effects it is expected that the values of figures of merit would initially increase with microgrid size, up until the point when there is an adequate level of DER flexibility and capacity, beyond which increasing the microgrid size would result in the saturation of the figures of merit, and no significant further improvement to CPF control. The simulation results presented in this paper comply with this expectation. For small microgrids less than 10 dwellings as there are insufficient resources to dispatch, the CPFC algorithm is generally incapable of controlling $\mathrm{CPF}$ at the target. This is also reflected by comparatively lower improvement to the values of figures of merit for such microgrids. It is however important to note that the control effectiveness and the values of figures of merit increase as the microgrid size is increased from 2 to 10 dwellings. By increasing the microgrid size from
0 to 30 dwellings, the control capability of the CPFC algorithm is improved further, and the values of figures of merit increase however at a reduced rate. The values of the figures of merit start to saturate when the microgrid size is increased beyond 30 dwellings as the CPF is effectively controlled at its target value $P F_{T}$. Based on above observations it is possible to conclude that for the system under investigation in this study, the recommended microgrid size threshold is at 30 dwellings. Reducing the microgrid size below this threshold results in less improvements to the effectiveness of the CPFC algorithm and increasing the microgrid size beyond this limit does not generally result in better control of the microgrid power flow. Having established the relationship between the effectiveness of the CPFC and the Microgrid size, in order to improve the control effectiveness even further, it is necessary to either increase the device flexibility or storage capacity of the DERs. Since DER flexibility is left to the discretion of the user it cannot be predicted with absolute certainty. On the other hand the incorporation of sufficient storage capacity for DERs with an inherent storage element such as a large enough hot water storage tank in a GSHP system, or the inclusion of optimally sized bulk battery within the Microgrid is expected to result in further improvement of the CPF control. However it is crucial to size the DER storage capacity appropriately with respect to both the Microgrid size and CPFC's requirements. A novel methodology based on the spectral analysis of the Microgrid's residual energy for determining the DER storage capacity threshold has been devised by the authors and will be presented in a future publication.

\section{REFERENCES}

E. E. Limited, "Electric vehicles in the UK and Republic of Ireland: Greenhouse gas emission reductions \& infrastructure needs," Element Energy LimitedAugust 2010.

[2] P. Papadopoulos, L. M. Cipcigan, N. Jenkins, and I. Grau, "Distribution networks with Electric Vehicles," in Universities Power Engineering Conference (UPEC), 2009 Proceedings of the 44th International, 2009, pp. 1-5.

[3] T. Ackermann and V. Knyazkin, "Interaction between distributed generation and the distribution network: operation aspects," in Transmission and Distribution Conference and Exhibition 2002: Asia Pacific. IEEE/PES, 2002, pp. 1357-1362 vol.2.

[4] I. Richardson, M. Thomson, D. Infield, and C. Clifford, "Domestic electricity use: A high-resolution energy demand model," Energy and Buildings, vol. 42, pp. 1878-1887, 2010.

[5] A. Fazeli , Sumner, M, Johnson, C M, Christopher, E, "Coordinated Optimal Dispatch of Distributed Energy Resources within a Smart Energy Com-munity Cell," presented at the 2012 3rd IEEE PES Innovative Smart Grid Technologies Europe Berlin, 2012.

[6] A. W. M. J. v. S. a. M. H. M. d. Wit, "ADVANCED SIMULATION OF BUILDING SYSTEMS AND CONTROL WITH SIMULINK," presented at the Eighth International IBPSA Conference, 2003.

[7] P. S. Anca D.Hansen, Lars H. Hansen and Henrik Bindner, "Models for a Stand-Alone PV System," Riso National Laboratory, RoskildeDecember 2000.

[8] N. W. Miller, "Dynamic modeling of GE 1.5 and 3.6 MW wind turbine-generators for stability simulations," presented at the Power Engineering Society General Meeting, 2003, IEEE, 2003.

[9] R. X. a. J. F. Hongwen He *, "Evaluation of Lithium-Ion Battery Equivalent Circuit Models for State of Charge Estimation by an Experimental Approach," Energies, vol. 4, 2011.

[10] M. W. Gangel and R. F. Propst, "Distribution Transformer Load Characteristics," Power Apparatus and Systems, IEEE Transactions on, vol. 84, pp. 671-684, 1965. 